\title{
Polyaniline Modified Stainless Steel Fiber Felt for High-Performance Microbial Fuel Cell Anodes
}

\author{
Junxian Hou, Zhongliang Liu, and Yanxia Li
}

\begin{abstract}
The three-dimensional (3D) macroporous anodes are constructed by coating polyaniline(PANI) on stainless steel fiber felts (SSFFs) that have an open, solid and macroporous structure. In the present study, nano-polyaniline is synthesized in two different methods by using chemical and electrochemical oxidative polymerization. These modified electrodes provide large surface area for reaction, interfacial transport and biocompatible interface available for bacterial colonization and substrate transport. The polarization curves together with electrochemical impedance spectroscopy (EIS) measurements demonstrate that polyaniline modified anodes could greatly improve MFCs' power output and decrease MFCs' internal resistance. Our experimental results also prove that embedding polyaniline into 3D macroporous metallic scaffold is a promising method for MFC anode fabrication.
\end{abstract}

Index Terms-Microbial fuel cell, anode, polyaniline, stainless steel fiber felt, polymerization.

\section{INTRODUCTION}

Microbial fuel cells (MFCs) convert chemical energy into electrical energy directly via electro-active micro-organisms, and have a great potential application for simultaneous wastewater treatment and energy recovery which is the main advantage over other conventional wastewater treatment methods [1], [2]. However, the relatively low power density of MFCs resulted from the large energy loss remains one of the main unsolved obstacles for their practical applications. The total energy loss in the MFC system can be understood from the voltage equation, $V=E_{t}-\eta_{\text {ohmic }}-\eta_{\text {act }}-\eta_{\text {conc }}$, in which $\eta_{\text {act }}, \eta_{\text {ohmic }}$ and $\eta_{\text {conc }}$ are voltage losses due to ohmic polarization, reaction kinetics and mass transport, respectively [3], [4]. Research has showed that activated loss and diffusion resistance accounting for more than $70 \%$ of the total resistance of MFCs [3], [4]. An effective way for removing these difficulties is to employ macroporous electrodes and nano-materials (macro- and nano-structure). The 3D macroporous based-electrode can serve as macroporous scaffolds for reaction, interfacial transport and dispersion of active sites at different length scales of pores and shorten diffusion paths or reduce diffusion resistance effect [5]. Nano-materials such as grapheme (GN), carbon nanotube (CNT), polymer and their composites modified macroporous scaffolds can provide large surface area for reaction and have been proved efficient in minimizing the anode energy loss in the MFC system and significantly

Manuscript received March 30, 2014; revised June 30, 2014. This work was financially supported by the Beijing Municipal Science and Technology Plan Project (D14110900270000).

The authors are with the College of Environmental and Energy Engineering, Beijing University of Technology, Beijing 100124, China (e-mail: liuzhl@bjut.edu.cn, hjx@emails.bjut.edu.cn). increasing the MFC power density output [6], [7].

Polyaniline (PANI) is a useful conducting polymer that has been widely used for electronic, optical and electrochemical applications owing to its low cost, good environmental stability, electroactivity, high pseudocapacitance and unusual doping/dedoping chemistry [8], which can be readily synthesized by either chemical oxidation or electrochemical oxidative polymerization of aniline. As we all know, PANI has been widely used in MFC anode and cathode and demonstrated that it can effectively improve MFCs' performance. The results of Ghasemi et.al showed that polyaniline modifications increased the microorganism's adhesion, which play a significant role as biocatalysts and so improved the performance of MFCs [9]. Lai et al. [10] modified carbon cloth by electrochemical polymerization of aniline as MFC anode. The result showed that the maximum power density $\left(5.16 \mathrm{~W} / \mathrm{m}^{3}\right)$ of the modified electrode achieved 2.66 times higher than that of the unmodified.

Our previous study has demonstrated that carbon nanoparticles modified three-dimensional macroporous AISI 316L stainless steel fiber felts (SSFF) can greatly decrease the polarization resistance and shows good electrochemical kinetics compared with carbon-base electrodes [11]. The benefits of using stainless steel fiber felt are many, such as high conductivity, high strength and being ease in incorporating a flow field. Most important of all, it has a three-dimensional macroporous structure which can make the biofilm be accessible even deep into the interior of the anode, and thus the majority of the interior structure is effectively used. In this paper, we fabricate the MFC anodes by using polyaniline nanoparticles to modified SSFFs. Polyaniline nanoparticles are synthesized by using chemical and electrochemical polymerization, respectively. The power curves and electrochemical impedance spectroscopy (EIS) demonstrate that these two types of modified electrodes greatly increase the MFCs' power output, resulting from its good 3D macroporous structure combined the macro-SSFF scaffold with the nano-polyaniline nanoparticles which enhance the interaction between the biofilm and electrode, simultaneously increase anode reactive surface area.

\section{THE EXPERIMENTAL}

\section{A. Electrode Fabrication}

The stainless steel fiber felt (Xi'an Filter Metal Materials Co., Ltd, China) substrates were cut into $1.8 \mathrm{~cm} \times 1.8 \mathrm{~cm}$ pieces, and were soaked in acetone for $3 \mathrm{~h}$ to dissolve the organic substances adsorbed and then rinsed with ultrapure water before the experiments.

An electrochemical cell with a three electrode configuration was employed for electropolymerization. The 
electrochemical experiments were performed by using the CHI660D (CH Instruments, Shanghai). Stainless steel fiber felt (SSFF, $1.8 \mathrm{~cm} \times 1.8 \mathrm{~cm}$ ) was used as working electrode, a $\mathrm{Pt}$ mesh was used as counter electrode and $\mathrm{Ag} / \mathrm{AgCl}(3.5 \mathrm{M}$ $\mathrm{KCl}$ ) served as reference electrode. PANI was electropolymerized on SSFFs for 35 cycles scanned back and forth from $-0.2 \mathrm{~V}$ to $+0.8 \mathrm{~V}$ versus $\mathrm{Ag} / \mathrm{AgCl}$ at a scan rate of $10 \mathrm{mV} / \mathrm{s}$ in $0.5 \mathrm{M} \mathrm{H}_{2} \mathrm{SO}_{4}$ aqueous solution containing $0.1 \mathrm{M}$ aniline. In this way, the PANI/SSFF anodes were obtained and denoted as $\mathrm{PANI}_{\text {ele }} / \mathrm{SSFF}$ in which the figure in the subscript indicates the electropolymerization cycle number. After each experiment, the remaining electrolyte on the electrode surface was rinsed with deionized water and then dried naturally in the air.

Chemical polymerization of PANI modified SSFF was prepared by immersing SSFFs in the PANI aqueous solution obtained above for $12 \mathrm{~h}$, dried naturally in the air, then obtained $\mathrm{PANI}_{\text {che }} / \mathrm{SSFF}$ electrode. PANI was synthesized by a rapid mixing reaction. The purified aniline $(0.3 \mathrm{~g}, 3.2 \mathrm{mmol})$ was dissolved in $10 \mathrm{~mL}$ of $1 \mathrm{M} \mathrm{HCl}$ aqueous solution. While maintaining vigorous stirring at room temperature, ammonium peroxydisulfate $(0.18 \mathrm{~g}, 0.8 \mathrm{mmol})$ in $10 \mathrm{~mL}$ of 1 $\mathrm{M} \mathrm{HCl}$ aqueous solution was poured into the aniline solution rapidly. Chemical polymerization was observed in about 5 min when the characteristic green color of polyaniline emeraldine salt appeared. The mixture was stirred at room temperature overnight and then rinsed with the ultrapure water until it is neutral $(\mathrm{pH}=7)$ and dried naturally in the air before use [6].

\section{B. MFC Setup and Operation}

An H-shaped two-chamber MFC was constructed $(6 \mathrm{~cm}$ deep, $15 \mathrm{~cm}^{2}$ of the cross sectional area) for these experiments. The two chambers were separated by a cation exchange membrane (Ultex, Membranes International, Inc., USA), and the volume of both the chambers was $90 \mathrm{~mL}$. MFC reactors were inoculated with a mixed bacterial culture from the anode compartment of another H-shaped MFC, which was originally inoculated with domestic wastewater from Beijing Gaobeidian Wastewater Treatment Plant and was operated for more than half a year utilizing acetate.

The cell voltage across the external resistance $\left(R_{e x t}=1000\right.$ $\Omega$ ) was recorded every $20 \mathrm{~min}$ using a data acquisition system (Agilent, USA). Three different anodes (SSFF,) were assembled in the same three reactors and ran in parallel, namely SSFF-MFC, PANI che $/ \mathrm{SSFF}$. PANI ele $/ \mathrm{SSFF}$. Carbon felts $(1 \mathrm{~cm} \times 2 \mathrm{~cm}$, Beiing Sanye Co., Ltd, China) were chosen as the cathode of these MFCs. The anolyte consisted of $1 \mathrm{~g} / \mathrm{L}$ $\mathrm{CH}_{3} \mathrm{COONa}, \quad 0.3 \mathrm{~g} / \mathrm{L} \quad \mathrm{NH}_{4} \mathrm{Cl}, \quad 1 \mathrm{~g} / \mathrm{L} \quad \mathrm{NaCl}, 0.04 \mathrm{~g} / \mathrm{L}$ $\mathrm{CaCl}_{2} \cdot 2 \mathrm{H}_{2} \mathrm{O}, 0.3 \mathrm{~g} / \mathrm{L} \mathrm{MgSO}_{4}, 0.2 \mathrm{~g} / \mathrm{L} \mathrm{NaHCO}_{3}, 10.7 \mathrm{~g} / \mathrm{L}$ $\mathrm{K}_{2} \mathrm{HPO}_{4}$ and $5.3 \mathrm{~g} / \mathrm{L} \mathrm{KH}_{2} \mathrm{PO}_{4}$. The cathodic chambers of all the three MFCs were filled with $50 \mathrm{mM}$ ferricyanide and 100 $\mathrm{mM}$ phosphate buffer solution $\left(10.7 \mathrm{~g} / \mathrm{L} \mathrm{K}_{2} \mathrm{HPO}_{4}, 5.3 \mathrm{~g} / \mathrm{L}\right.$ $\mathrm{KH}_{2} \mathrm{PO}_{4}, \mathrm{pH}=6.9$ ) [6], [7]. The MFCs were operated under the fed-batch mode condition and the anolyte was replaced after the voltage below $50 \mathrm{mV}$. In the third circle when operation, power density curves and polarization curves were obtained by changing the circuit external resistor from 9999 $\Omega$ to $50 \Omega$. All experiments were carried out in a $33 \pm 0.5^{\circ} \mathrm{C}$ incubator with a $1000 \Omega$ external resistance connected unless otherwise specified. The power density was normalized based on the projected anodic surface area.

\section{Characterization}

Electrochemical impedance spectroscopy (EIS) was employed to measure the total internal resistances of the MFCs, and the measurements were conducted using the CHI660D (CH Instruments, Shanghai) at the working potential while the MFCs were operated with a constant external resistance $\left(R_{\text {ext }}=1000 \Omega\right)$. Two electrode experiments were performed on the whole cell with the anode as the working electrode and the cathode used as the counter and reference electrode in a frequency of $100 \mathrm{kHz}$ to $5 \mathrm{mHz}$ with an AC signal amplitude of $10 \mathrm{mV}$ [7], [12]-[14]. SEM (Hitachi S-4300N) was employed to characterize the surface morphologies of the samples.

\section{RESULTS AND DISCUSSION}

\section{A. Morphology Analysis}

Fig. 1 shows the SSFF morphologies before and after polyaniline modification. One can see that the surface of SSFF is smooth when without modification, as displayed in Fig. 1(a) and Fig. 1(b). SSFF is an ideal substrate for fabrication of electrodes used for the MFCs due to its solid, highly conductive, and 3D macroporous structure. The drawback of the SSFF is that its biocompatibility is not as good as that of the carbon-base anodes, which results in a limited bacterial colonization on the surface of fibers. To solve this problem, in the present study, polyaniline (PANI) was used to modify the fibers of SSFFs aiming at improving the biocompatibility and decreasing its overpotential as well as increasing the specific surface area accessible for the bacteria colonization. Fig. 1(c) and Fig. 1(d) display the morphologies of $\mathrm{PANI}_{\text {che }} / \mathrm{SSFF}$, the polyaniline modified SSFFs by using the chemical oxidative polymerization method. Fig. 1(e) and Fig. 1(f) display the morphologies of $\mathrm{PANI}_{\text {ele }} / \mathrm{SSFF}$, the polyaniline modified SSFFs by using the electrochemical oxidative polymerization method. The modified SSFFs have a wrinkled and crumpled structure that makes the fiber surfaces rough and micro-structuralized. One can clearly observe that the modified electrodes have a good nano- and macro-structure on the macro-porous scaffold of the three-dimensional (3D) macro-SSFF for the attachment of nano-polyaniline. What is more, the conductive polyaniline coating accords with the felt morphology without changing its open porous structure, so the macropores of the resulting electrodes could allow bacteria colonization in the inner structure of the 3D electrode, and thus enhance the interaction between the biofilm and the electrode.

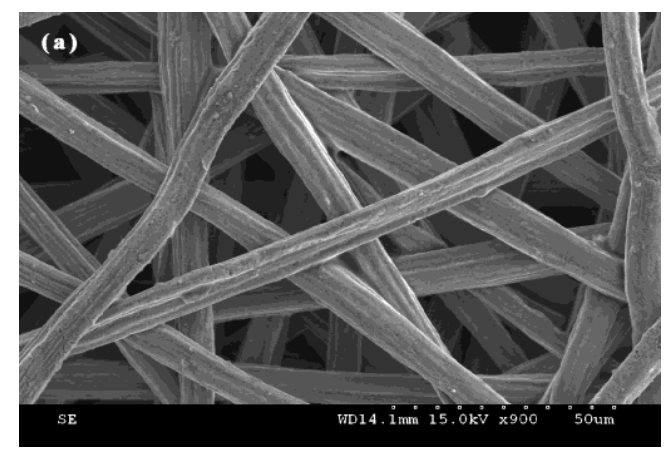



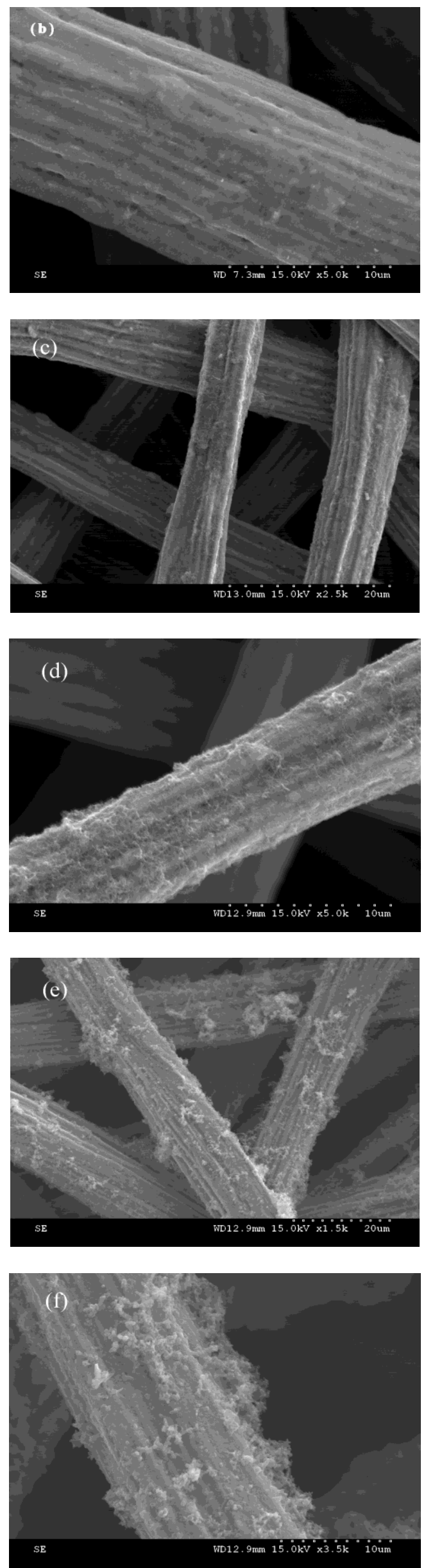

Fig. 1. SEM images of the SSFFs before and after polyaniline modification in different magnification, (a)(b)SSFF, (c)(d)PANI che $_{\text {/SSFF, (e)(f) }}$ PANI $\mathrm{ele}_{\mathrm{le}} / \mathrm{SSFF}$

\section{B. Power Curve}

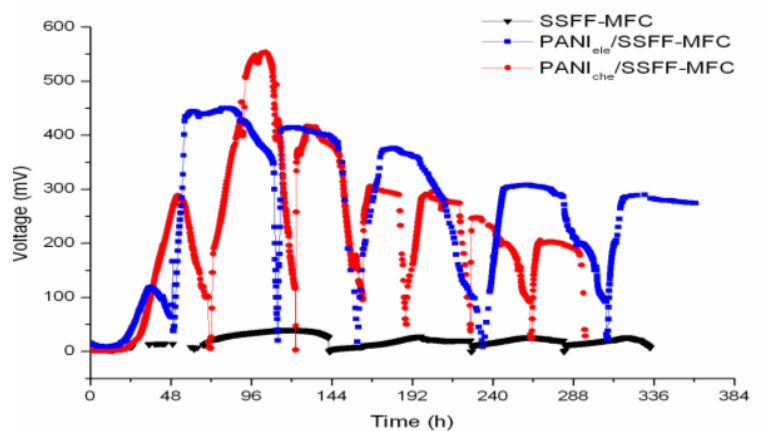

Fig. 2. Voltage output of the SSFF-, PANI $\mathrm{che}_{\text {che }} / \mathrm{SSFF}$ - and $\mathrm{PANI}_{\text {ele }} / \mathrm{SSFF}-\mathrm{MFC}$ after inoculation.

To investigate the full-cell performance of PANI modified SSFF electrodes, three MFC reactors (SSFF-MFC, $\mathrm{PANI}_{\text {che }} / \mathrm{SSFF}$ and $\mathrm{PANI}_{\mathrm{ele}} / \mathrm{SSFF}-\mathrm{MFC}$ ) were run in parallel with the same inoculum but different anodes. As shown in Fig. 2, MFCs with the SSFF, PANI ${ }_{\text {che }} / \mathrm{SSFF}$ and $\mathrm{PANI}_{\text {ele }} / \mathrm{SSFF}$ as the anode reached a maximum voltage of $25 \mathrm{mV}, 552 \mathrm{mV}$ and $451 \mathrm{mV}$ respectively, after 2-3 days operation. It should be noted that, as shown in this figure, the unmodified SSFF-MFC simply did not work. The reasons are many, but the following are among of the most important. As we have pointed out above, the stainless steel is less efficient for interfacial electron transfer, thus it requires a large overpotential [7], [11]. Pocaznoi's experimental results showed that although the microbial anodes formed on stainless steel gave a higher current than those formed on flat graphite, but the stainless steel was revealed to be not able to ensure electron transfer that was as fast as that of graphite or carbon cloth [7], [11]. Furthermore, the stainless steel's biocompatibility is not as good as that of the carbon-based electrodes. Thus, the number of bacteria growing on the fibers is very limited and the reactive area of SSFF is small. All these will result in a low electron transfer rate. However, after the polyaniline modification, the performance of $\mathrm{PANI}_{\text {che }} / \mathrm{SSFF}$ and PANI $\mathrm{ele}_{\mathrm{el}} / \mathrm{SSFF}$ all has a great improvement. The reason is that these two types of methods synthesized polyaniline nanoparticles modified electrodes offered a desirable integration of the low overpotential, the biocompatible surface and the large specific surface area, and these desirable characteristics make the charge transfer at the interface become easier. It should be also noted, the maximum voltage of each cycle gradually declined for every MFC as the operation time goes by. This maybe result from the clogging of the 3D macropores or/and the decrease of PANI redox activities.

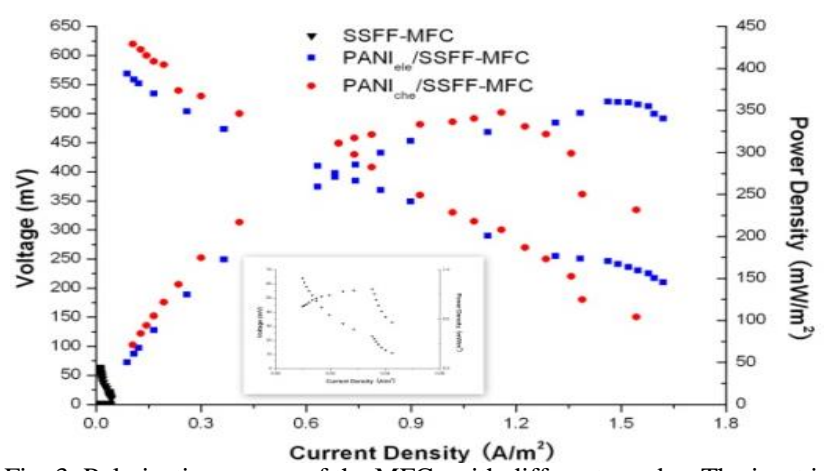

Fig. 3. Polarization curves of the MFCs with different anodes. The inset is the enlarged polarization curves of the SSFF-MFC. 
The polarization curves and power density curves are illustrated in Fig. 3, they were all obtained in the third circle when operation. From Fig. 3, one can see that the power generation is greatly improved after electrode modification, likely due to the excellent properties of polyaniline nanoparticles. To be specific, the maximum power density of the $\mathrm{PANI}_{\text {che }} / \mathrm{SSFF}-\mathrm{MFC}$ is $347 \mathrm{~mW} / \mathrm{m}^{2}$ at a current density of $1.15 \mathrm{~A} / \mathrm{m}^{2}$, while the $\mathrm{PANI}_{\text {ele }} / \mathrm{SSFF}-\mathrm{MFC}$ achieves its maximum power density of $360 \mathrm{~mW} / \mathrm{m}^{2}$ at the current density of $1.49 \mathrm{~A} / \mathrm{m}^{2}$. For the unmodified SSFF-MFC, the maximum power density is only $0.8 \mathrm{~mW} / \mathrm{m}^{2}$ at the current density of $0.035 \mathrm{~A} / \mathrm{m}^{2}$. This may be due to the high overpotential and the poor biocompatibility of the SSFF, as we have discussed earlier. In summary, these two types of electrodes all can greatly improve MFC performance. The reason is that an excellent 3D macroporous electrode structure can be ensured by integrating the good electro-activity and biocompatibility of the polyaniline nanoparticles with the high conductivity and large macropores of the SSFF scaffold. Although SSFFs could not be directly used as MFCs' anode, coating a polyaniline layer of a proper thickness may take both the advantage of both PANI and SSFF and this may offer us exciting opportunities in the advancement of MFC anode design.

\section{Electrochemical Analysis}

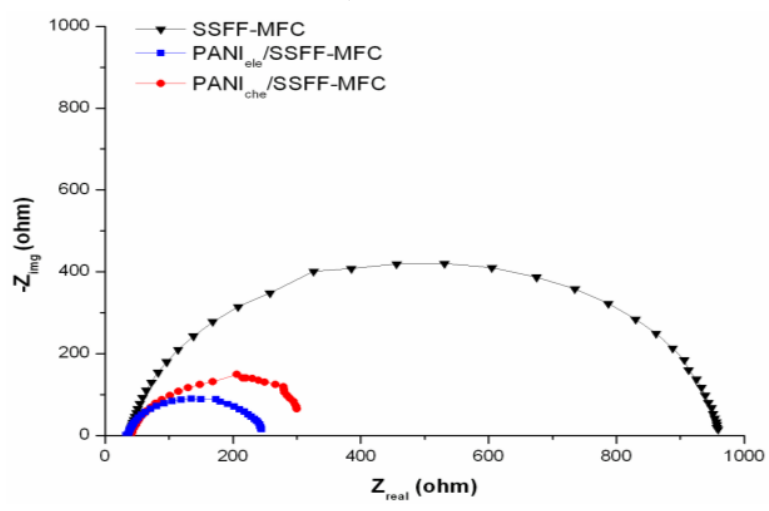

Fig. 4. EISs of the SSFF-, PANI che $/ S S F F-$ and $\mathrm{PANI}_{\text {ele }} / \mathrm{SSFF}-\mathrm{MFC}$ under working cell conditions using two-electrode mode. The inset is the enlarged EIS curves.

The better electrochemical behaviors and kinetics of the anodes enable the higher output power density of the MFCs. To understand the electrochemical effect of these modified anodes on the total resistance of the MFCs better, two-electrode mode EIS experiments are conducted for all the MFCs under working cell condition $\left(R_{e x t}=1000 \Omega\right)$. After the steady-state current generation achieved, the Nyquist plots are obtained and shown in Fig. 4. Impedance studies of MFCs' total resistance with different anodes often use a two time-constant model for resistance estimation [7], [12]-[14]. The equivalent circuit $R_{1}\left(R_{2} Q\right)\left(R_{3} C\right)$ is used here to calculate the MFCs' total resistance, in which $R_{l}$ represents the Ohmic resistance, and $R_{2}$ represents the charge transfer resistance, $R_{3}$ and $C$ in parallel represent the finite diffusion [7], [12]-[14]. By fitting the data of the Nyquist plots, the estimated total resistance of the $\mathrm{PANI}_{\mathrm{che}} / \mathrm{SSFF}-\mathrm{MFC}$ and $\mathrm{PANI}_{\text {ele }} / \mathrm{SSFF}-\mathrm{MFC}$ is $315.6 \Omega$ and $248.3 \Omega$, respectively, which are all much smaller than that of the unmodified SSFF-MFC whose total resistance is calculated to be $974.5 \Omega$. The measured ohmic resistance $R_{s}$ for the SSFF-MFC,
$\mathrm{PANI}_{\text {che }} / \mathrm{SSFF} \mathrm{MFC}$, and PANI $\mathrm{ele}_{\text {ele }} / \mathrm{SSFF}-\mathrm{MFC}$ is $36.1 \Omega, 36.5$ $\Omega$ and $32.7 \Omega$, respectively. Therefore, the polarization resistance of the $\mathrm{PANI}_{\text {che }} / \mathrm{SSFF}-\mathrm{MFC}$ and $\mathrm{PANI}_{\text {ele }} / \mathrm{SSFF}-\mathrm{MFC}$ is $279.1 \Omega$ and $215.6 \Omega$, respectively, while that of the SSFF-MFC is $938.4 \Omega$. The EIS results demonstrate that these two type of polyaniline nanoparticles on anodes can greatly decrease the polarization resistance and have good electrochemical kinetics and of course can produce a higher power output. It also confirms that coating polyaniline nanoparticles onto metallic scaffold is a promising and effective method for anode fabrication.

\section{CONCLUSION}

Polyaniline nanoparticles synthesized by using chemical and electrochemical oxidative polymerization methods modified stainless steel fiber felts as MFC anodes offers a desirable integration of the low overpotential, the biocompatible surface and the high electrochemical activity, which makes the charge transfer at the interface easier. The experimental results show that these modified electrodes can greatly increase the power output and low the polarization resistance. The reason for this may be the fact that the excellent macro- and nano-structure of the anode is developed which takes the advantage of the 3D macroporous scaffold of SSFFs and the excellent electroactivity of nano-polyaniline. This study also proves that a thin polyaniline layer of a proper thickness combined with the SSFF is a promising and effective method for the fabrication of high-performance anodes.

\section{ACKNOWLEDGMENTS}

This work is supported by the Beijing Municipal Science and Technology Plan Project (D14110900270000).

\section{REFERENCES}

[1] B. E. Logan and K. Rabaey, "Conversion of wastes into bioelectricity and chemicals by using microbial electrochemical technologies," Science, vol. 337, pp. 686-690, Aug. 2012.

[2] A. L. Guerreroa, I. M. Headc, F. Mateoa, A. Ginestaa, and C. Godineza, "Effect of temperature on the performance of microbial fuel cells," Fuel, vol. 89, pp. 3985-3994, June 2010.

[3] P. Y. Zhang and Z. L. Liu, "Experimental study of the microbial fuel cell internal resistance," J. Power Sources, vol. 195, pp. 8013-8018, Dec. 2010.

[4] Q. Wen, Y. Wu, L. X. Zhao, and Q. Sun, "Production of electricity from the treatment of continuous brewery wastewater using a microbial fuel cell," Fuel, vol. 89, pp. 1381-1385, July 2010.

[5] Y. Li, Z. Y Fu, and B. L. Su, "Hierarchically structured porous materials for energy conversion and storage," Adv. Funct. Mater., vol. 22, pp. 4634-4667, Nov. 2012.

[6] J. X. Hou, Z. L. Liu, and P. Y. Zhang, "A new method for fabrication of graphene/polyaniline nanocomplex modified microbial fuel cell anodes," J. Power Sources, vol. 224, pp. 139-144, Feb. 2013.

[7] J. X. Hou, Z. L. Liu, S. Q. Yang, and Y. Zhou, "Three-dimensional macroporous anodes based on stainless steel fiber felt for high-performance microbial fuel cells," J. Power Sources, vol. 258, pp. 204-209, July 2014.

[8] L. Wang, X. P. Lu, S. B. Lei, and Y. H. Song, "Graphene-based polyaniline nanocomposites: Preparation, properties and applications," J. Mater. Chem. A, vol. 2, pp. 4491-4509, 2014.

[9] M. Ghasemi, W. Ramli, W. Daud et al., "The effect of nitric acid, ethylenediamine, and diethanolamine modified polyaniline nanoparticles anode electrode in a microbial fuel cell," Int. J. Hydrogen Energy, vol. 38, pp. 9525-9532, July 2013.

[10] B. Lai, X. H. Tang, H. R. Li, Z. W. Du, X. W. Liu, and Q. Zhang, "Power production enhancement with a polyaniline modified anode in 
microbial fuel cells," Biosens. Bioelectron., vol. 28, pp. 373-377, Oct. 2011.

[11] D. Pocaznoi, A. Calmet, L. Etcheverry, B. Erable, and A. Bergel, "Stainless steel is a promising electrode material for anodes of microbial fuel cells," Energy Environ. Sci., vol. 5, pp. 9645-9652, Sep. 2012.

[12] Y. Ahn and B. E. Logan, "Altering anode thickness to improve power production in microbial fuel cells with different electrode distances," Energy Fuels, vol. 27, pp. 271-276, 2013.

[13] Y. Yin, G. T. Huang, Y. R. Tong, Y. D. Liu, and L. H. Zhang, "Electricity production and electrochemical impedance modeling of microbial fuel cells under static magnetic field," J. Power Sources, vol. 237, pp. 58-63, Sep. 2013.

[14] A. J. Hutchinson, J. C. Tokash, and B. E. Logan, "Analysis of carbon fiber brush loading in anodes on startup and performance of microbial fuel cells," J. Power Sources, vol. 196, pp. 9213-9219, Nov. 2011.

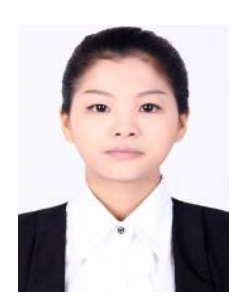

Junxian Hou is a Ph.D. candidate of the College of Environmental and Energy Engineering, Beijing University of Technology, China. She received her B.S. degree from Changchun Institute of Technology, China, in 2010. Then obtained her M.S. degree from Beijing University of Technology, China, in 2013. Her main research interests are microbial fuel cell electrode materials and transport phenomena.

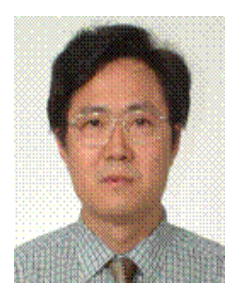

Zhongliang Liu is a professor in thermal fluids and energy engineering of the College of Environmental and Energy Engineering of Beijing University of Technology, China. He received his B.S. degree from Huadong Petroleum Institute, China, in 1982. He then obtained his M.S. degree from Dalian Marine College, China, in 1984. From 1987 to 1988 , he worked in the Mechanical Engineering Department of Birmingham University as a visiting scholar. In 1996, he received his Ph.D. degree from the Power Engineering Department of Southeast University, China. His main research interests include environmental energy technologies, renewable energy and energy conservation theory and technology, enhanced heat transfer, numerical methods in heat transfer and fluid flow.

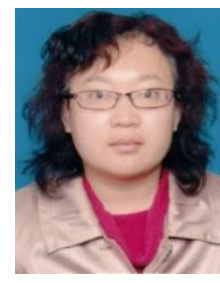

Yanxia $\mathbf{L i}$ is a lecturer of the College of Environmental and Energy Engineering, Beijing University of Technology, China. She received her B.S. degree from Shandong University, China, in 2000. She then obtained her M.S. degree from Beijing University of Technology, China, in 2003. Since 2003, she worked in the College of Environmental and Energy Engineering, Beijing University of Technology, China. In 2012, she received her Ph.D. degree from Beijing University of Technology, China. Her main research interests include enhanced heat transfer, small-scale catalytic combustion and energy conservation theory and technology. 